\title{
Pulmonary valve and atrial lead infective endocarditis: A successful non-surgical treatment of significant vegetation with pulmonary complications
}

\author{
Michał Majewski ${ }^{1}$, Andrzej Kułach ${ }^{1}$, Anna Drzewiecka ${ }^{2}$, \\ Rafał Gardas ${ }^{2}$, Krzysztof S. Gołba ${ }^{2}$, Zbigniew Gąsior ${ }^{1}$ \\ ${ }^{1}$ Department of Cardiology, School of Health Sciences, Medical University of Silesia, Katowice, Poland \\ ${ }^{2}$ Department of Electrocardiology and Heart Failure, School of Health Sciences, \\ Medical Univesity of Silesia, Katowice, Poland
}

A 64-year-old male with congestive heart failure and an implantable cardioverter-defibrillator in place with a 3-month history of febrile, fatigue, cough, and weight loss was referred with a suspicion of cardiac device-related infective endocarditis which was confirmed with a blood culture (Streptococcus gallolyticus).

Transthoracic echocardiography (TTE) and transesophageal echocardiography showed reduced left ventricular ejection fraction (25\%), moderate tricuspid regurgitation with no features of vegetation, floating masses on the pulmonary valve (PV) causing functional $\mathrm{PV}$ stenosis with severe regurgitation (Vmax $2.1 \mathrm{~m} / \mathrm{s}$, PHT $228 \mathrm{~ms}$; Fig. 1A, B), and an oscillating mass on the atrial lead (Fig. 1C). Cardiac computed tomography (CT) revealed a widening of the pulmonary trunk with emboli at the bifurcation. The patient received empirical (vancomycin, gentamicin) and subsequently targeted (vancomycin, ciprofloxacin) antibiot- ic therapy, followed by hardware removal with transvenous lead extraction (Libertor locking stylet and Byrd Sheath; Cook Vacsular Inc, USA; Fig. 1E). The patient had not qualified for PV surgery due to high procedural risk.

After 3 weeks, peripheral pulmonary embolism was still observed in an angio-CT, along with lesions suspected for malignancy in both lungs (Fig. 1F), which was excluded by high-resolution CT and bronchofiberoscopy. At 3-month followup there was no fever, inflammatory markers were low, pulmonary lesions were resolved and TTE showed stable PV vegetation size.

We described a rare case of lead-related infective endocarditis with co-existing PV vegetations and no tricuspid valve involvement. Complete hardware removal and antimicrobial therapy turned out to be a sufficient treatment option. Large vegetations with numerous pulmonary emboli can be effectively treated non-surgically if a patient is hemodynamically stable.

Conflict of interest: None declared

Address for correspondence: Dr. Andrzej Kułach, Department of Cardiology, School of Health Sciences, Medical University of Silesia in Katowice, ul. Ziołowa 47, 40-635 Katowice, Poland, tel: +48505863793, e-mail: andrzejkulach@gmail.com

Received: $16.06 .2020 \quad$ Accepted: 12.10 .2020

This article is available in open access under Creative Common Attribution-Non-Commercial-No Derivatives 4.0 International (CC BY-NC-ND 4.0) license, allowing to download articles and share them with others as long as they credit the authors and the publisher, but without permission to change them in any way or use them commercially. 


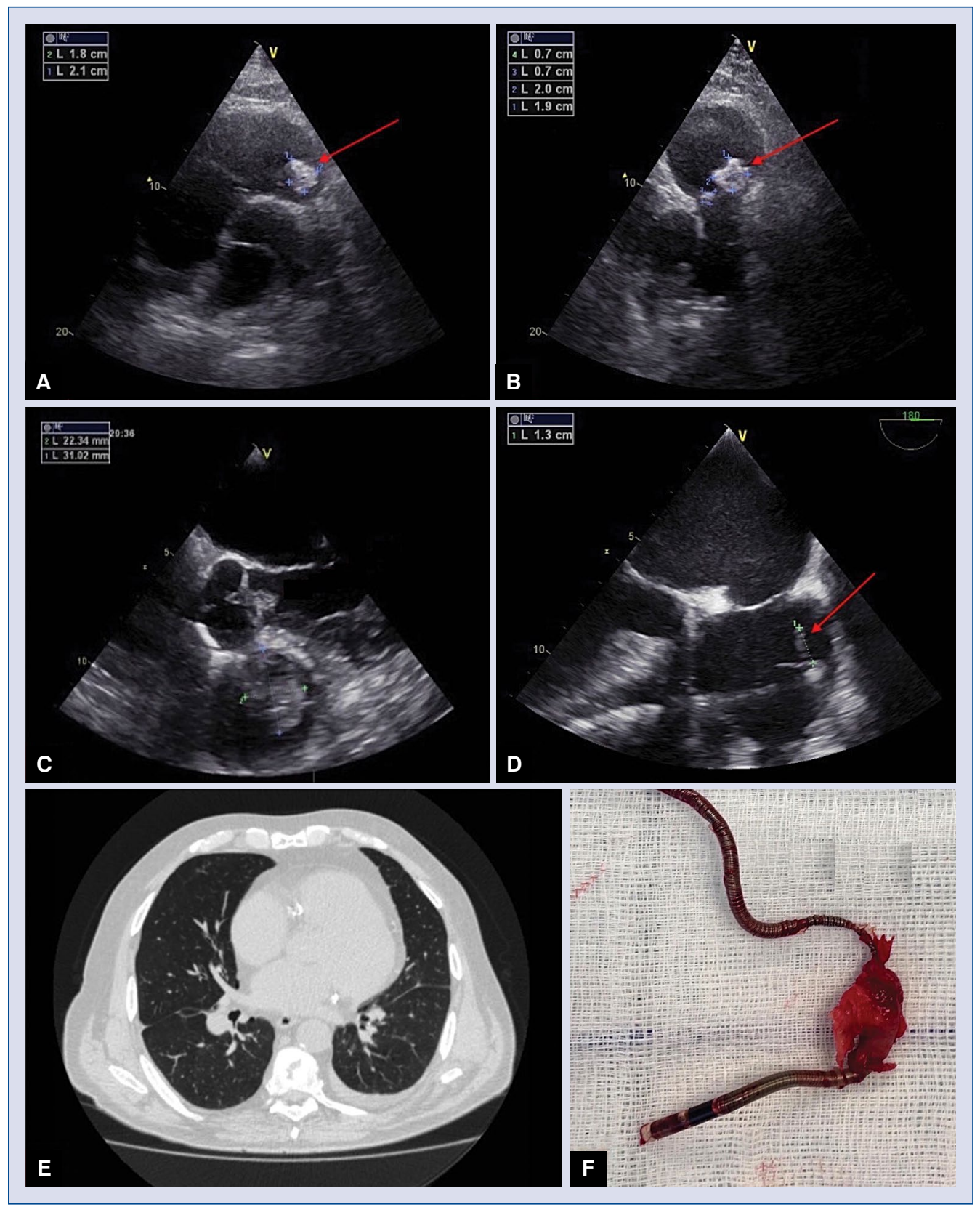

Figure 1. A. Two-dimensional transthoracic echocardiography (2D TTE) parasternal short-axis view at the level of the great vessels: vegetation is visible at the pulmonary artery valve (main pulmonary artery focused projection); B. 2D TTE focused on the pulmonary valve; C. Large vegetation $(22 \times 31 \mathrm{~mm}$ in size $)$ in right ventricular outflow tract seen in transesophageal echocardiography (TEE); D. TEE showing atrial lead vegetation; E. Computed tomography scan showing multiple bilateral lung opacities mimicking malignancy that turned out to be inflammatory; F. Atrial lead vegetation (intraoperative photography). 